ISSN 0103-9954

\title{
RIQUEZA, DIVERSIDADE E COMPOSIÇÃO FLORÍSTICA EM ÁREAS DE CERRADO EM REGENERAÇÃO E PRESERVADO NA ESTAÇÃO ECOLÓGICA DE ITIRAPINA - SP
}

\author{
RICHNESS, DIVERSITY, AND FLORISTIC COMPOSITION IN REGENERATING AND \\ PRESERVED ‘CERRADO’ AREAS IN ITIRAPINA ECOLOGICAL STATION, SÃO PAULO STATE
}

\author{
Alessandro de Paula ${ }^{1}$ Fernanda Quintas Martins ${ }^{2}$ Marco Antônio Portugal Luttembarck Batalha ${ }^{3}$ \\ Rodrigo Rodrigues ${ }^{4}$ Marco Antônio Manhães ${ }^{5}$
}

\section{RESUMO}

A capacidade das espécies do cerrado de se regenerar a partir da rebrota de estruturas subterrâneas depende tanto das propriedades físicas e químicas do solo quanto do tempo decorrido após o desmatamento. Na Estação Ecológica de Itirapina, realizam-se levantamentos florísticos e fitossociológicos dos componentes herbáceo-subarbustivo e arbustivo-arbóreo da vegetação de cerrado em uma área em regeneração, após dois anos da remoção de um talhão de Pinus elliotti, e em uma área preservada. Em cada uma das duas áreas, foram lançadas, ao longo de uma transecção, 20 parcelas de $5 \times 5 \mathrm{~m}$, distribuídas sistematicamente a cada $20 \mathrm{~m}$. Nessas parcelas foi amostrado o componente arbustivo-arbóreo (todos os indivíduos lenhosos com diâmetro do caule no nível do solo igual ou maior a $3 \mathrm{~cm}$ ). Em cada uma das parcelas foi delimitada uma subparcela de $0,5 \times 0,5 \mathrm{~m}$, em que foi amostrado o componente herbáceo-subarbustivo (todos os indivíduos lenhosos com diâmetro do caule no nível do solo menor do que $3 \mathrm{~cm}$ e todos os não lenhosos). O menor índice de diversidade encontrado foi para o componente herbáceo-subarbustivo da área em regeneração $\left(1,29\right.$ nats.indivíduo $\left.{ }^{-1}\right)$, enquanto que o maior foi para o componente herbáceo-subarbustivo da área de cerrado preservado $\left(2,75\right.$ nats.indivíduo $\left.{ }^{-1}\right)$. Entre as áreas em regeneração e preservada, os índices de similaridade de Sørensen, tanto para o componente herbáceo-subarbustivo $(0,23)$ quanto para o componente arbustivo-arbóreo (0,31), mostraram uma baixa semelhança florística entre as áreas. Além disso, a densidade e a riqueza para ambos os componentes foram significativamente maiores para o cerrado preservado. Os resultados encontrados mostraram que os dois anos decorridos desde o corte raso de Pinus elliotti não foram suficientes para que a vegetação natural se restabelecesse no talhão.

Palavras-chave: savana; fitossociologia; Pinus elliotti.

\section{ABSTRACT}

The capacity of 'cerrado' species to regrow from underground organs after deforestation depends on the soil physical and chemical properties and the time elapsed since the disturbance. In Itirapina Ecological

1 Engenheiro Florestal, Dr., Professor Adjunto do Departamento de Engenharia Agrícola e Solos, Universidade Estadual do Sudoeste da Bahia, Estrada do Bem Querer, Km 4, Caixa Postal 95, CEP 45083-900, Vitória da Conquista (BA), Brasil. apaula@uesb.edu.br

2 Bióloga, MSc., Professora do Centro Universitário Leonardo da Vinci, Rodovia BR 470, 1040, Benedito, CEP 89130-000, Indaial (SC), Brasil. fernanda_quintas@uol.com.br

3 Biólogo, Dr., Professor Associado do Departamento de Botânica, Universidade Federal de São Carlos, Via Washington Luís, Km 235, Caixa Postal 676, CEP 13565-905, São Carlos (SP), Brasil. marcobat@uol.com.br

4 Biólogo, Discente do Programa de Pós-Graduação em Ecologia e Recursos Naturais, Universidade Federal de São Carlos, Via Washington Luís, Km 235, Caixa Postal 676, CEP 13565-905, São Carlos (SP), Brasil. rods2br@yahoo.com.br

5 Biólogo, Dr., Téenico-administrativo do Instituto de Ciências Biológicas, Universidade Federal de Juiz de Fora, Rua José Lourenço Kelmer, Bairro São Pedro, CEP 36036-900, Juiz de Fora (MG), Brasil. marcomanhaes@bol.com.br 
Station, we carried out floristic and phytosociological surveys of both herbaceous and woody components of the vegetation in 'cerrado' in a regenerating plot, after two years of Pinus elliotti removal, and in a neighboring preserved the 'cerrado' area. In each of the two areas have been launched along a transection, 20 parts of $5 \mathrm{~m}$ x $5 \mathrm{~m}$, systematically distributed to each $20 \mathrm{~m}$. These plots were sampled the woody component (all woody plants with stem diameter at ground level equal to or greater than $3 \mathrm{~cm}$ ). In each of the plots was defined a sub-portion of $0.5 \mathrm{~m} \times 0.5 \mathrm{~m}$, which was sampled at the herbaceous component (all woody plants with a stem diameter at the ground level lower than $3 \mathrm{~cm}$ and all non-woody). The lowest diversity index was found for the herbaceous component of the regeneration area (1.29 nats.individual $\left.{ }^{-1}\right)$, while the highest was for the herbaceous component of the preserved area of 'cerrado' ( 2.75 nats. individual $\left.{ }^{-1}\right)$. Among the areas being regenerated and preserved, Sørensen's similarity indices for both the herbaceous component $(0.23)$ and for the woody component $(0.31)$, showed low floristic similarity between areas. Besides, the density and richness for both components were significantly higher for the area preserved in 'cerrado'. The results showed that the two years elapsed since Pinus elliotti clear-cut were not enough for the natural vegetation recovery.

Keywords: Brazilian savanna; phytosociology; Pinus elliotti.

\section{INTRODUÇÃO}

No Brasil, o Domínio do Cerrado ocupa aproximadamente dois milhões de $\mathrm{km}^{2}$, o que representa cerca de $23 \%$ do território nacional (RATTER et al., 1997), sendo considerada a segunda maior província fitogeográfica do país em área (RIBEIRO e WALTER, 1998). As principais ameaças à sua biodiversidade estão relacionadas a duas atividades econômicas: a monocultura intensiva de grãos e a pecuária extensiva de baixa tecnologia (WWF-PRO-CER, 1995). Além dessas atividades, áreas de cerrado têm sido convertidas em silviculturas de Eucalyptus spp. e Pinus spp. Durante as três ultimas décadas, cerca de $2.000 \mathrm{~km}^{2}$ de cerrado já foram transformados em plantações de Pinus spp. (ESPÍRITO SANTO, 1995). Apenas $1,47 \%$ das áreas originais de cerrado estão protegidas na forma de unidades de conservação de uso indireto (BRASIL, 1998); daí, a necessidade de se conservar e recuperar os remanescentes existentes nesse domínio.

As espécies de cerrado apresentam, geralmente, alto potencial de regeneração natural a partir de estruturas subterrâneas (RIZZINI e HERINGER, 1962; GOODLAND e FERRI, 1979; HOFFMANN, 1998). Embora haja evidências científicas de que a reprodução a partir de sementes é mecanismo importante para a regeneração de algumas espécies (FELIPE e SILVA, 1984; FRANCO et al., 1996; OLIVEIRA e SILVA, 1993), a regeneração por brotação tem maior êxito no processo de recuperação da cobertura vegetal do cerrado (DURIGAN et al., 1998). Essa capacidade peculiar das espécies do cerrado de recobrir o terreno a partir da rebrota de estruturas subterrâneas depende das propriedades físicas e químicas do solo e do tempo decorrido após o desmatamento (DURIGAN et al., 1998).

Em um modelo simplificado para áreas degradadas, podem-se dividir as espécies, independentemente de sua origem, em pioneiras (crescimento rápido) e não pioneiras (crescimento mais lento), que, em um processo de sucessão natural, as primeiras são mais eficientes em explorar substratos desnudos e em suportar condições de microclima desfavoráveis (CORREA e CARDOSO, 1998). As espécies de estádios sucessionais posteriores encontram um substrato física, química e biologicamente diferenciado e, com isso, possuem vantagens competitivas (CORREA e CARDOSO, 1998).

Os estudos de regeneração da vegetação nativa em áreas agrícolas, inicialmente desmatadas e abandonadas após o uso, concluíram que o processo de revegetação natural inicia-se com a reprodução de espécies cujas sementes ali chegaram, germinaram e as plantas se estabeleceram (ODUM, 1988). Características locais, como distância de bancos de sementes, presença de substâncias tóxicas no substrato abandonado, textura, instabilidade da superfície e camadas mais profundas, compactação, retenção de água, $\mathrm{pH}$, salinidade, declividade, entre outras, determinam as espécies aptas a se estabelecerem, além de facilitarem ou dificultarem o processo de recolonização (CORRÊA e LEITE, 1998). À medida que a área vai sendo recolonizada, há uma sucessão de espécies, um acúmulo de 
biomassa vegetal, um aumento da biodiversidade, até que, ao final dessa sequência, uma comunidade, semelhante à original, domina o local (ODUM, 1988).

Este trabalho teve como objetivo comparar a estrutura da comunidade vegetal em duas áreas de cerrado, uma em regeneração há dois anos desde a retirada de um talhão de Pinus elliotti Engelm. - e outra preservada. Para isso, procurouse responder às seguintes perguntas: a riqueza, a diversidade e a densidade de plantas vasculares no cerrado preservado são maiores do que no cerrado em regeneração? Qual é a similaridade florística entre essas duas áreas? A proporção entre espécies herbáceo-subarbustivas e arbustivo-arbóreas é a esperada para áreas de cerrado?

\section{MATERIAL E MÉTODO}

Este trabalho foi desenvolvido na Estação Ecológica de Itirapina (EEI), pertencente ao Instituto Florestal do Estado de São Paulo e localizada nos municípios de Itirapina e Brotas (aproximadamente,

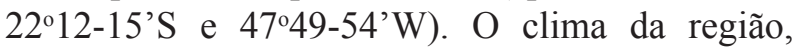
segundo a classificação de Köppen (1948), é do tipo Cwa, isto é, mesotérmico úmido de inverno seco com precipitação anual média de $1.459 \mathrm{~mm}$, concentrada no período de outubro a março (ZANCHETTA, 2006). A área total da reserva é de aproximadamente 2.300 ha, sendo que as fisionomias observadas correspondentes à vegetação natural são cobertura vegetal predominantemente constituída por campos, campos cerrados, florestas ripárias e áreas úmidas (ZANCHETTA e DINIZ, 2006).

O levantamento foi realizado em duas áreas adjacentes (aproximadamente, entre 22 ${ }^{\circ} 12^{\prime} 31$ $37^{\prime}$ 'S e 47053'53-54'W), uma não perturbada e outra em regeneração: a primeira era coberta por campo cerrado (Figura 1), enquanto que a segunda era coberta por um cerrado em regeneração há dois anos, quando um talhão de Pinus elliotti existente no local foi removido por corte raso (Figura 2). Em cada uma das duas áreas, foram lançadas ao longo de uma transecção, 20 parcelas de 5 x $5 \mathrm{~m}$, distribuídas sistematicamente a cada $20 \mathrm{~m}$. Nessas parcelas, foi amostrado o componente arbustivo-arbóreo, definido aqui como todos os indivíduos lenhosos com diâmetro do caule no nível do solo igual ou maior a $3 \mathrm{~cm}$, conforme recomendado por SMA (1997). Em cada uma das parcelas, foi delimitada uma subparcela de $0,5 \times 0,5 \mathrm{~m}$, em que foi amostrado o componente herbáceo-subarbustivo, definido aqui como todos os indivíduos lenhosos com diâmetro do caule no nível do solo menor do que $3 \mathrm{~cm}$ e todos os não lenhosos. Nesse componente, para as gramíneas e ciperáceas, considerou-se cada touceira como um indivíduo.

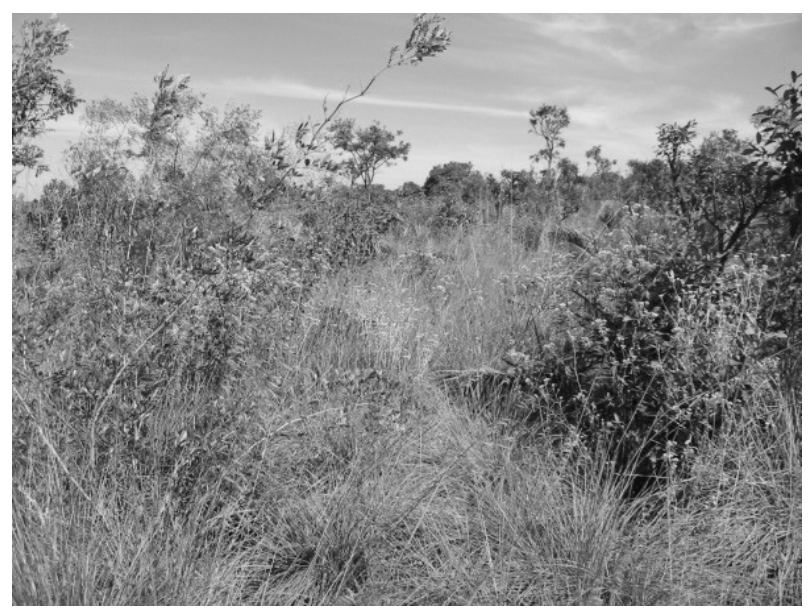

FIGURA 1: Vista parcial da área de campo cerrado preservado estudado na Estação Ecológica de Itirapina (Itirapina, São Paulo).

FIGURE 1: Partial view of the preserved area of 'campo cerrado' studied in the Ecological Station of Itirapina (Itirapina, São Paulo state).

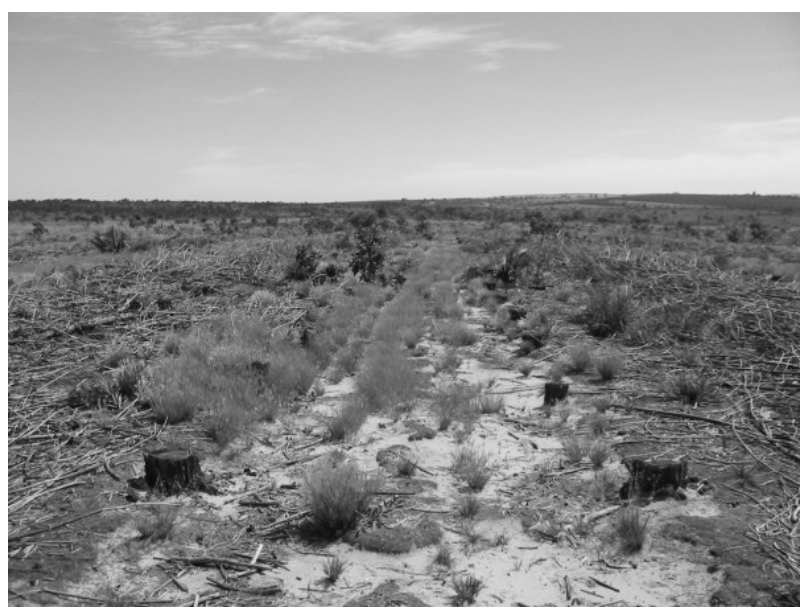

FIGURA 2: Vista parcial da área em regeneração estudada na Estação Ecológica de Itirapina (Itirapina, São Paulo).

FIGURE 2: Partial view of the area regeneration studied in the Ecological Station of Itirapina (Itirapina, São Paulo state. 
Foram identificados os indivíduos amostrados em campo com o auxílio de uma chave de identificação baseada em caracteres vegetativos (BATALHA e MANTOVANI, 1999). Para apresentação da listagem florística foi adotado o sistema APG III (2009). Para cada um dos componentes, foram calculados os seguintes descritores fitossociais: riqueza, densidade e diversidade. A riqueza foi calculada como o número de espécies por unidade amostral e a diversidade pelo índice de Shannon-Weaver (MAGURRAN, 1988). A densidade foi estimada segundo MuellerDombois e Ellenberg (1974). A proporção entre espécies herbáceo-subarbustivas e arbustivoarbóreas foi comparada com aquela esperada por Mantovani e Martins (1993) para áreas de cerrado com o teste de qui-quadrado (ZAR, 1999). Também foi comparada essa proporção entre os cerrados em regeneração e preservado.

Foi testada a normalidade das distribuições das variáveis riqueza e densidade com o teste de Kolmogorov-Smirgov (ZAR, 1999). Com o objetivo de testar se as diferenças entre as áreas de cerrado em regeneração e preservado eram significativas, foi utilizado o teste $t$ não pareado (ZAR, 1999) para a riqueza e densidade dos componentes herbáceosubarbustivo e componente arbustivo-arbóreo, uma vez que todas as variáveis apresentaram distribuições normais. Foi calculado o poder de cada teste $t$ (ZAR, 1999), para verificar a probabilidade de se rejeitar a hipótese nula quando ela fosse falsa. Para testar se as diferenças entre os índices de diversidade foram significativas, foi aplicado o teste de Hutcheson (1970). Também foi calculado o índice de similaridade de Sørensen (MAGURRAN, 1988) entre ambos os componentes das áreas em regeneração e preservada.

\section{RESULTADOS E DISCUSSÃO}

Nas 40 parcelas amostradas, foram encontradas 56 espécies, distribuídas em 50 gêneros e 27 famílias (Tabela 1). A família mais representativa foi Fabaceae, com oito espécies (ou $14 \%$ do total), seguida de Poaceae e Myrtaceae, com seis espécies $(11 \%)$ cada. As famílias mais bem representadas nesta amostragem estão entre as mais ricas também para outras áreas de cerrado (e.g., MANTOVANI e MARTINS, 1993; BATALHA et al., 1997; BATALHA e MARTINS, 2002; MUNHOZ e FELFILI, 2006). Já Tannus e Assis (2004), também estudando uma área de campo sujo no município de Itirapina, encontraram como as famílias mais importantes Asteraceae, Fabaceae e Poaceae.

A família Poaceae foi a que apresentou o maior número de indivíduos (112) dentre os 328 amostrados, perfazendo $34 \%$ do total. As duas espécies mais abundantes foram gramíneas, Loudetiopsis chrysothrix (Nees) Conert, com 34 indivíduos, e Gymnopogon foliosus (Willd.) Ness, com 33 indivíduos.

Foram amostradas 32 espécies no componente herbáceo-subarbustivo, das quais cinco eram indivíduos jovens de espécies arbustivoarbóreas e 29 espécies no componente arbustivoarbóreo. Assim, das 56 espécies que amostradas no total, 27 foram herbáceo-subarbustivas e 29 arbustivo-arbóreas. Logo, a proporção entre espécies herbáceo-subarbustivas e arbustivo-arbóreas no levantamento foi de $0,93: 1$, valor significativamente diferente $\left(\chi^{2}=5,86 ;\right.$ g.1. $\left.=1 ; p=0,016\right)$ do mínimo encontrado por Mantovani e Martins (1993) para áreas de cerrado, que é de 2:1. No cerrado em regeneração, a razão entre espécies herbáceosubarbustivas e arbustivo-arbóreas foi de 0,89:1 e, no cerrado preservado, essa razão foi de 0,93:1. Essas duas proporções não foram significativamente diferentes entre si $\left(\chi^{2}=0,17\right.$; g.1. $\left.=1 ; p=0,683\right)$.

Quatro das 27 espécies herbáceosubarbustivas amostradas ocorreram nos dois tipos de cerrado, bem como cinco das 29 espécies arbustivoarbóreas. Dessa forma, o índice de similaridade de Sørensen para o componente herbáceo-subarbustivo da área em regeneração e da área preservada foi de 0,23 e para o componente arbustivo-arbóreo, 0,31.

Para o componente herbáceo-subarbustivo, na área de cerrado em regeneração, foi encontrado 0,85 (média) $\pm 0,93$ (desvio padrão) espécie. 0,25 $\mathrm{m}^{-2}$, enquanto que na área de cerrado preservado, o valor foi de $2,40 \pm 3,97$ espécies. $0,25 \mathrm{~m}^{-2}$. As duas médias foram significativamente diferentes entre si $(\mathrm{t}=5,88 ;$ g.l. $=38 ; \mathrm{p}<0,001)$. Para o componente arbustivo-arbóreo, na área de cerrado em regeneração, foi encontrado 0,60 $\pm 0,94$ espécie. $25 \mathrm{~m}^{-2}$, enquanto que na área de cerrado preservado, o valor foi de $3,25 \pm 1,33$ espécies. $25 \mathrm{~m}^{-2}$, médias significativamente diferentes $(\mathrm{t}=7,26 ; \mathrm{g} .1 .=38 ; \mathrm{p}$ $<0,001)$. Nos dois casos, o poder do teste foi de $100 \%$.

Quanto à densidade, foram encontrados $2,40 \pm 3,97$ indivíduos. $0,25 \mathrm{~m}^{-2}$ para o componente herbáceo-subarbustivo da área de cerrado em regeneração, enquanto que, na área de cerrado 
TABELA 1: Lista das espécies encontradas nas áreas em regeneração e de campo cerrado da Estação Ecológica de Itirapina (Itirapina, São Paulo), em que "N" = número de indivíduos da espécie e "Componente e Área" = 1 - componente herbáceo-subarbustivo da área em regeneração, 2 componente arbustivo-arbóreo da área em regeneração, 3 - componente herbáceo-subarbustivo da área preservada e 4 - componente arbustivo-arbóreo da área preservada.

TABLE 1: List of species found in regenerating areas, and "campo cerrado" Ecological Station Itirapina (Itirapina, São Paulo), where "N" = number of individuals of the species and "Component and Area" = 1 - herbaceous component of the area regeneration, 2 - woody component of the area regeneration, 3 - herbaceous component of the conservation area and 4 - the woody component of the area preserved.

\begin{tabular}{|c|c|c|c|}
\hline Família & Espécie & $\mathrm{N}$ & Componente e Área \\
\hline Annonaceae & Annona crassiflora Mart. & 2 & 4 \\
\hline Apocynaceae & Aspidosperma tomentosum Mart. & 5 & 4 \\
\hline Arecaceae & Attalea geraensis Barb. Rodr. & 2 & 3 \\
\hline Arecaceae & Syagrus petraea (Mart.) Becc. & 9 & 3 \\
\hline Asteraceae & Baccharis rufescens Spreng. & 1 & 4 \\
\hline Asteraceae & Eupatorium squalidum DC. & 2 & 3 \\
\hline Asteraceae & Gochnatia barrosii Cabrera & 2 & 3 \\
\hline Asteraceae & Trichogonia salviifolia Gardner & 3 & 3 \\
\hline Bignoniaceae & Jacaranda caroba (Vell.) A. DC. & 3 & 4 \\
\hline Bignoniaceae & Jacaranda decurrens Cham. & 1 & 1 \\
\hline Bignoniaceae & Tabebuia ochracea (Cham.) Standl. & 21 & 3,4 \\
\hline Calophyllaceae & Kielmeyera coriacea Mart. \& Zucc. & 2 & 4 \\
\hline Caryocaraceae & Caryocar brasiliense Cambess. & 4 & 2,4 \\
\hline Celastraceae & Peritassa campestris (Cambess.) A.C. Sm. & 2 & 1 \\
\hline Chrysobalanaceae & Couepia grandiflora (Mart. \& Zucc.) Benth. ex Hook f. & 3 & 2 \\
\hline Chrysobalanaceae & Parinari excelsa Sabine & 2 & 3 \\
\hline Connaraceae & Connarus suberosus Planch. & 2 & 4 \\
\hline Convolvulaceae & Merremia digitata (Spreng.) Hallier f. & 2 & 1 \\
\hline Cyperaceae & Bulbostylis junciformis (Kunth) C.B. Clarke & 4 & 1 \\
\hline Cyperaceae & Bulbostylis sphaerocephala (Boeck.) C.B. Clarke & 4 & 1,3 \\
\hline Ebenaceae & Diospyros hispida A. DC. & 3 & 4 \\
\hline Erythroxylaceae & Erythroxylum suberosum A. St.-Hil. & 6 & 2,4 \\
\hline Fabaceae & Acosmium subelegans (Mohlenbr.) Yakovlev & 19 & 4 \\
\hline Fabaceae & Bauhinia rufa (Bong.) Steud. & 2 & 2 \\
\hline Fabaceae & Chamaecrista basifolia (Vogel) H.S. Irwin \& Barneby & 1 & 3 \\
\hline Fabaceae & Dalbergia miscolobium Benth. & 9 & 2,4 \\
\hline Fabaceae & Dimorphandra mollis Benth. & 2 & 4 \\
\hline Fabaceae & Machaerium acutifolium Vogel & 1 & 4 \\
\hline Fabaceae & Senna rugosa (G. Don) H.S. Irwin \& Barneby & 2 & 3 \\
\hline Fabaceae & Stryphnodendron obovatum Benth. & 1 & 2 \\
\hline Indeterminada & Indeterminada & 1 & 3 \\
\hline Lamiaceae & Aegiphila lhotzkiana Cham. & 5 & 2,4 \\
\hline Malpighiaceae & Banisteriopsis stellaris (Griseb.) B. Gates & 3 & 3 \\
\hline Malpighiaceae & Byrsonima coccolobifolia Kunth & 3 & 4 \\
\hline Malpighiaceae & Byrsonima intermedia A. Juss. & 1 & 3 \\
\hline
\end{tabular}


TABELA 1: Continuação ...

TABLE 1: Continued ...

\begin{tabular}{|c|c|c|c|}
\hline Família & Espécie & $\mathrm{N}$ & Componente e Área \\
\hline Malvaceae & Eriotheca gracilipes (K. Schum.) A. Robyns & 1 & 4 \\
\hline Melastomataceae & Miconia albicans (Sw.) Steud. & 6 & 3,4 \\
\hline Melastomataceae & Miconia ligustroides (DC.) Naudin & 2 & 4 \\
\hline Myrtaceae & Campomanesia pubescens (DC.) O. Berg & 10 & 3,4 \\
\hline Myrtaceae & Eugenia hiemalis Cambess. & 1 & 4 \\
\hline Myrtaceae & Eugenia punicifolia (Kunth) DC. & 5 & $2,3,4$ \\
\hline Myrtaceae & Myrcia guianensis (Aubl.) DC. & 4 & $1,3,4$ \\
\hline Myrtaceae & Myrcia lasiantha DC. & 1 & 2 \\
\hline Myrtaceae & Psidium australe Cambess. & 1 & 1 \\
\hline Poaceae & Aristida jubata (Arechav.) Herter & 8 & 3 \\
\hline Poaceae & Brachiaria decumbens Stapf & 16 & 1,3 \\
\hline Poaceae & Gymnopogon foliosus (Willd.) Ness & 33 & 1,3 \\
\hline Poaceae & Loudetiopsis chrysothrix (Nees) Conert & 34 & 3 \\
\hline Poaceae & Panicum repens $\mathrm{L}$. & 17 & 3 \\
\hline Poaceae & Tristachya leiostachya Nees & 4 & 3 \\
\hline Polypodiaceae & Polypodium latipes Langsd. \& L. Fisch. & 5 & 3 \\
\hline Primulaceae & Rapanea guianensis Aubl. & 1 & 4 \\
\hline Rubiaceae & Alibertia sessilis (Vell.) K. Schum. & 10 & 3 \\
\hline Sapindaceae & Talisia angustifolia Radlk. & 3 & 3 \\
\hline Sapotaceae & Pouteria torta (Mart.) Radlk. & 19 & 4 \\
\hline Sapotaceae & Pradosia brevipes (Pierre) T.D. Penn. & 12 & 3 \\
\hline
\end{tabular}

preservado, o valor foi de 7,50 \pm 3,97 indivíduos. $0,25 \mathrm{~m}^{-2}$. As duas médias foram significativamente diferentes $(\mathrm{t}=3,85 ; \mathrm{g} .1 .=38 ; \mathrm{p}<0,001)$. Já para $\mathrm{o}$ componente arbustivo-arbóreo, foram encontrados $0,75 \pm 1,44$ indivíduos. $25 \mathrm{~m}^{-2}$ no cerrado em regeneração e $5,80 \pm 3,10$ indivíduos. $25 \mathrm{~m}^{-2}$ no cerrado preservado, valores significativamente diferentes $(t=6,59$, g. $1 .=38, p<0,001)$. Em ambos os casos, os poderes do teste foram de $100 \%$.

Os valores de diversidade para o componente herbáceo-subarbustivo do cerrado em regeneração e do cerrado preservado foram, respectivamente, de $1,29 \pm 0,04$ nats. ind $^{-1}$ e 2,75 $\pm 0,02$ nats. ind ${ }^{-1}$, valores estes significativamente diferentes entre si $(\mathrm{t}=34,43 ; \mathrm{g} .1 .=75 ; \mathrm{p}<0,001)$. Para o componente arbustivo-arbóreo, esses valores foram de 2,08 \pm 0,14 nats. ind ${ }^{-1}$ para o cerrado em regeneração e 2,68 $\pm 0,02$ nats. ind ${ }^{-1}$ para o cerrado preservado, valores também significativamente diferentes entre si $(\mathrm{t}=4,30 ;$ g.l. $=16 ; \mathrm{p}<0,001)$.

Os índices de similaridade de Sørensen entre as áreas em regeneração e preservada, tanto para o componente herbáceo-subarbustivo quanto para o componente arbustivo-arbóreo, mostraram uma baixa semelhança entre as mesmas, ainda que contíguas uma a outra.

O componente herbáceo-subarbustivo de flora de cerrado é mais rico e mais vulnerável do que o componente arbustivo-arbóreo (BATALHA e MARTINS, 2002). Em áreas de cerrado, a proporção entre espécies herbáceo-subarbustivas e arbustivoarbóreas varia de 2:1 a 3:1 (MANTOVANI e MARTINS, 1993). No levantamento em questão, a proporção geral, incluindo as duas áreas, foi menor do que o mínimo estabelecido por Mantovani e Martins (1993). Vale ressaltar que Tannus e Assis (2004) encontraram uma relação de 3,6:1 do componente herbáceo-subarbustivo em relação ao arbustivo-arbóreo, resultado esse que demonstra potencialmente a proporção em uma área preservada.

Essas proporções demonstram uma redução da riqueza das espécies herbáceo-subarbustivas nos dois ambientes, mas especialmente na área em recuperação. A elevada abundância da planta invasora Brachiaria decumbens na área de cerrado preservado pode ser a responsável 
pelo empobrecimento do componente herbáceosubarbustivo, reduzindo o estabelecimento das espécies herbáceo-subarbustivas. Tannus e Assis (2004), já destacaram que a presença de manchas de Brachiaria decumbens estaria desfavorecendo a diversidade da flora nativa no Cerrado.

Em relação à área em regeneração, foi amostrada uma única touceira de Brachiaria decumbens. É possível a ocorrência de alguma forma de inibição ao desenvolvimento de Brachiaria decumbens, seja essa inibição química ou física, devido à grande quantidade de matéria orgânica acumulada sobre o solo após a operação de corte e desrama de Pinus elliotti e sua disposição em leiras. $\mathrm{O}$ acúmulo dessa matéria orgânica sobre o solo apresenta vantagens, tais como a diminuição do efeito da erosão no solo logo após o desmatamento e a inibição da Brachiaria decumbens e desvantagens, como a inibição ao estabelecimento das espécies autóctones do cerrado, a diminuição da velocidade de recobrimento do solo e o consequente aumento da erosão a longo prazo. Zanchetta e Diniz (2006) recomendaram a retirada das espécies exóticas da Estação Ecológica de Itirapina no intuito de preservar a vegetação nativa e reduzir os efeitos de sua contaminação biológica.

Os valores observados para o índice de Shannon-Weaver (H'), entre 1,91 e 2,75 nats. indivíduos $^{-1}$, demonstraram uma baixa diversidade nas áreas em regeneração e preservada. Mantovani (1998) relatou valores do índice de diversidade de Shannon-Weaver para sítios de cerrado de até 3,64 nats.indivíduos ${ }^{-1}$. Munhoz e Felfili (2006), por exemplo, encontraram valores variando de 3,0 a 3,2 nats.cobertura ${ }^{-1}$ em cinco períodos de amostragem em uma área de campo sujo no Distrito Federal. Já Batalha et al. (2001) encontraram valores de 3,46 nats.indivíduos $^{-1}$ para o componente herbáceosubarbustivo e de 3,74 nats.indivíduos ${ }^{-1}$ para o componente arbustivo-arbóreo.

\section{CONCLUSÕES}

Após dois anos, a estrutura do cerrado em regeneração é muito diferente do cerrado preservado. O perfil da vegetação da área em regeneração é, a princípio, distinto florística e estruturalmente daqueles das fases subsequentes da colonização, considerando a similaridade obtida com a área preservada. Assim, o processo de regeneração está se desenvolvendo com baixa riqueza, densidade e diversidade.

\section{AGRADECIMENTOS}

Ao Instituto Florestal do Estado de São Paulo (IF), pela cessão do alojamento, transporte e acesso à Estação Ecológica de Itirapina; aos funcionários do Instituto Florestal da Estação Ecológica de Itirapina, especialmente o Sr. Cláudio Correa Porto, a Sra. Denise Zanchetta, a Sra. Isabel e o Sr. José; ao doutorando Alexandro Marques Tozetti, pelo auxílio prestado durante os trabalhos de campo; e ao Programa de Pós-Graduação em Ecologia e Recursos Naturais da Universidade Federal de São Carlos, pelo transporte.

\section{REFERÊNCIAS BIBLIOGRÁFICAS}

APG. Angiosperm Phylogeny Group III. An update of the Angiosperm Phylogeny Group classification for the orders and families of flowering plants: APG III. Botanical Journal of the Linnean Society, v. 161, p. 105-121, 2009.

BATALHA, M. A.; MANTOVANI, W. Chaves de identificação das espécies vegetais vasculares baseada em caracteres vegetativos para a ARIE Cerrado Pé-de-Gigante (Santa Rita do Passa Quatro, SP). Revista do Instituto Florestal, v. 11, p. 137-158, 1999.

BATALHA, M. A.; ARAGAKI, S.; MANTOVANI, W. Florística do cerrado em Emas (Pirassununga, SP). Boletim de Botânica da Universidade de São Paulo, São Paulo, v. 16, p. 49-64, 1997.

BATALHA, M. A; MANTOVANI, W.; MESQUITA JR., H. N. Vegetation structure in cerrado physiognomies in southeastern Brazil. Brazilian Journal of Biology, v. 61, p. 475-483, 2001.

BATALHA, M. A; MARTINS, F. R. The vascular flora of the cerrado in Emas National Park (central Brazil). Sida, v. 20, p. 295-312, 2002.

BRASIL. Ministério do meio Ambiente, dos recursos hídricos e da Amazônica legal. Primeiro relatório nacional para a conservação sobre diversidade biológica. Brasília: Brasil, 1998. $235 \mathrm{p}$.

CORREA, R. S.; CARDOSO, E. S. Espécies testadas na revegetação de áreas degradadas. In: CÔRREA, R. S.; MELO FILHO, B. (Orgs.) Ecologia e recuperação de áreas degradadas no cerrado. Brasília: Paralelo 15, 1998, cap. 6, p. 101-116.

CORREA, R. S.; LEITE, L. L. Desmatamento e mineração em unidade de conservação. In: CÔRREA, R. S.; MELO FILHO, B. (Orgs.) 
Ecologia e recuperação de áreas degradadas no cerrado. Brasília: Paralelo 15, 1998, cap. 3, p. 29-47.

DURIGAN, G. et al. Indução do processo de regeneração da vegetação de cerrado em área de pastagem, Assis, SP. Acta Botanica Brasilica, v. 12, n. 3, p. 421-429, 1998.

ESPÍRITO SANTO, C. V. Diagnóstico e avaliação de setor florestal brasileiro região Centro-Oeste: Anuário executivo. Brasília: Funatura/Itto/Ibama, $1995.59 \mathrm{p}$.

FELIPE, G. M.; SILVA, J. C. S. Estudos de germinação em espécies de cerrado. Revista Brasileira de Botânica, v. 7, p. 157-163, 1984.

FRANCO, A. C.; NARDOTO, G. B.; SOUZA, M. P. Estabelecimento e crescimento de Dalbergia miscolobium Benth. em áreas de campo sujo e cerrado no DF. In: MIRANDA, H. S.; SAITO, C. H.; DIAS, B. F. S. (Orgs.) Impactos de queimadas em áreas de cerrado e restinga. Brasília: UnB, 1996, p. 84-92.

GOODLAND, R.; FERRI, M. G. Ecologia do cerrado. São Paulo: Itatiaia; Edusp, 1979. 193 p.

HOFFMAN, W. A. Post-burn reproduction of woody plants in a neotropical savanna: the relative importance of sexual and vegetative reproduction. Journal of Applied Ecology, v. 35, p. 422-433, 1998.

HUTCHESON, K. A test for comparing diversities based on Shannon formula. Journal of Theoretical Biology, v. 29, p. 151-154, 1970.

KÖPPEN, W. Climatología. México: Fondo de Cultura Económica, 1948. 478 p.

MAGURRAN, A. E. Ecological diversity and its measurement. Princeton: Princeton University, 1988. $179 \mathrm{p}$.

MANTOVANI, W. Methods for assessment of terrestrial phanerogams biodiversity. In: BICUDO, C. E. M.; MENEZES, N. A. (Eds). Biodiversity in Brazil. São Paulo: CNPq, 1998. p. 119-144.

MANTOVANI, W.; MARTINS, F. R. Florística do cerrado na reserva biológica de Moji Guaçu, SP. Acta Botanica Brasilica, v. 7, p. 33-60, 1993.

MUELLER-DOMBOIS, D.; ELLEMBERG, $\mathrm{H}$. Aims and methods of vegetation ecology. New York: John Willey \& Sons, 1974. 547 p.
MUNHOZ, C. B. R.; FELFILI, J. M. Fitossociologia do estrato herbáceo-subarbustivo de uma área de campo sujo no Distrito Federal, Brasil. Acta Botanica Brasilica, v. 20, n. 3, p. 671-685, 2006.

ODUM, E. P. Ecologia. Rio de Janeiro: Guanabara, 1988. $434 \mathrm{p}$.

OLIVEIRA, P. E.; SILVA, J. C. S. Reproductive biology of two species of Kielmeyera (Guttiferae) in the cerrados of Central Brasil. Journal of Tropical Ecology, v. 9, p. 67-79, 1993.

RATTER, J. A.; RIBEIRO, J. F.; BRIDGEWATER, $\mathrm{S}$. The Brazilian cerrado vegetation and threats to its biodiversity. Annals of Botany, v. 80, p. 223-230, 1997.

RIBEIRO, J. F.; WALTER, B. M. T. Fitofisionomias do bioma Cerrado. In: SANO, S. M.; ALMEIDA, S. P. (Eds). Cerrado: ambiente e flora. Planaltina: Embrapa, 1998. p. 89-166.

RIZZINI, C. T.; HERINGER, E. P. Studies on the underground organs of the trees and shrubs from some southern Brazilian savannas. Anais da Academia Brasileira de Ciências, v. 34, p. 235-247, 1962.

SMA SECRETARIA DO MEIO AMBIENTE. Cerrado: bases para conservação e uso sustentável das áreas de cerrado do Estado de São Paulo. São Paulo: SMA, 1997. 184 p.

TANNUS, J. L. S.; ASSIS, M. A. Composição de espécies vasculares de campo sujo e campo úmido em área de cerrado, Itirapina - SP, Brasil. Revista Brasileira de Botânica, v. 27, n. 3, p. 489-506, 2004.

ZANCHETTA, D.; DINIZ, F. V. Estudo da contaminação biológica por Pinus spp. em três diferentes áreas na Estação Ecológica de Itirapina (SP, Brasil). Revista do Instituto Florestal, v. 18, p. 1-14, 2006.

ZANCHETTA, D. (Coord.). Plano de manejo integrado das unidades de Itirapina $-1^{a}$ revisão. São Paulo: Secretaria do Meio Ambiente, Instituto Florestal, 2006. 318 p.

ZAR, J. H. Biostatiscal analysis. Upper Saddle River: Prentice Hall, 1999. 663 p.

WWF-PRO-CER. De grão em grão, o cerrado perde espaço (cerrado-impactos do processo de ocupação). Brasília: WWF, 1995. 115 p. 